\title{
WHEELED ROBOTS TO OVERCOME GROUND UNEVENNESS IN CONSTRUCTION AREAS
}

\author{
Kazuo Tani, Osamu Matsumoto, Shuuji Kajita, Nobumasa Shirai \\ Mechanical Engineering Laboratory \\ Namiki, Tsukuba, Ibaraki 305 Japan
}

\section{ABSTRACT}

The recent demands for use of robots in construction areas require realization of robots that can run and work over rough grounds. We seek for the technological possibilities to allow wheeled robots to move freely by overcoming unevenness of the ground. We have studied active suspensions for wheeled robots to minimize the body shake, a wheeled inverted pendulum to detect its attitude and the ground slope, and a wheeled jumping robot to pass over a step. Refining and combining these element technologies, we hope to realize useful wheeled terrain robots.

\section{INTRODUCTION}

Wheeled robots command their high-speed, efficient mobility on flat floors and are used in factories, hospitals, etc. But they often find difficulties in proceeding over uneven grounds, such as staircases indoors and rough terrain outdoors. It is an interesting theme of robotics how to provide wheeled robots with capabilities of overcoming unevenness while keeping their efficiency

We will have a lot of applications of such terrain robots: transport of materials and machines in construction sites, rescue and delivery in disaster areas, raising and harvesting in farms, orchards and forests, and so on. In any case the criteria for such robots will be: negotiable unevenness, running speed, payload, safety and stability, etc.

Many methods have been tried worldwide to materialize the functions of terrain robots. Active suspensions for cars have been studied to decrease body vibration and to improve manoeuvrability [1][2]. Articulated frames and suspensions for hillside tractors have been developed to maintain static stability of the body [3][4]. Leg-wheel systems have been devised to combine the advantages of legs and wheels [5][6].

The aim of our research is not to develop a readily practical terrain robot but rather to show possibilities of methods by studying and proposing mechanical and control elements. Emphasis is placed on introducing dynamic motions of unconventional mechanisms and on estimating the robot state using only internal sensors. We have studied active suspensions for wheeled robots to minimize the body shake, a wheeled inverted pendulum to detect its attitude and the ground slope, and a wheeled jumping robot to pass over a step. The results of our studies and experiments using
hardware models will be presented in the following chapters.

\section{ACTIVE SUSPENSION}

To enable a wheeled vehicle to overcome uneven grounds with stability of the body and the payload, the idea has been proposed of an active suspension which produces controlled force between the body and the wheel axle by means of an actuator. An active suspension is expected to play two kinds of roles: (1) to minimize the displacement and vibration of the vehicle body caused by the ground unevenness and (2) to enable the vehicle to pass over greater unevenness. In this chapter, we will discuss the results of our control experiments and simulations.

\subsection{Active suspension one-wheel model}

As the first step of our study of active suspensions, we built an experimental setup of an active suspension one-wheel model shown in Figure 1 [7]. It consists of two main parts: a vehicle
simulator and a road simulator.

The vehicle simulator consists of the body which slides vertically along the frame, the wheel which rides the road simulator, and the suspension which connects the body and the wheel. The suspension has, in parallel with coil springs which support the static weight of the body, a force- 
generating mechanism consisting of a direct-drive DC servo motor and a Chebyshev approximate straight line link mechanism. The link mechanism converts the torque produced by the motor into the force exerted between the body and the axle. It has a stroke of $240 \mathrm{~mm}$. It also works as a reduction mechanism with an equivalent arm length of $80 \mathrm{~mm}$ and is practically linear. The suspension displacement, or the body-axle distance, is measured with a rotary potentiometer attached to the motor. The vertical acceleration of the body is measured with a servo accelerometer.

The road simulator is made of a hydraulic cylinder to produce road profiles.

The reason why we used a direct-drive DC servo motor as the actuator of the suspension is The reason why we used a direct-drive
the simplicity of control in the experiment: it is comparatively linear, it is an almost pure force
generator, and it is back drivable. Force control is thus possible.

We used a computer M685 with CPU 68000 for the overall control of the system. It reads the sensor signals and controls the current of the motor. It also controls the road simulator.

The purpose of the suspension control is to suppress the vertical displacement of the vehicle body while the vehicle is excited by the road profile. Since the passive elements of the suspension and the friction can absorb the vibration in the high frequency domain, the role of the active suspension is to take care of the lower frequency domain.

We made a PID control system of the suspension in which the input signals are the vertical velocity of the body produced by analog integrating the accelerometer output and the suspension displacement obtained from the potentiometer. The signals are multiplied by the respective PID gains and summed up to produce the command to the motor. The gains are selected experimentally to minimize the vertical displacement of the body while the wheel is excited.

Note here that we restricted ourselves to the use of internal or inertial sensors (hereafter just internal sensors) for the control. Internal sensors do not have to look outside the robot to get information and it is very convenient and economical if we could do without external sensors.

Figure 2 shows some results of the experiments. A random road profile is given to the wheel. The body hardly moves vertically with control (a), but moves along the road profile without control (b), which proves that the control is working successfully.

2.2 Active suspension four-wheeled robot

Based on the result obtained using the one-wheel model, we built a four-wheeled robot with an independent active suspension for each wheel as shown in Figure 3 [8], wheelbase $800 \mathrm{~mm}$ and tread $540 \mathrm{~mm}$. We used the same suspension mechanism as the one-wheel model. The stiffness of the spring is $480 \mathrm{~N} / \mathrm{m}$. The maximum output torque of the motor is $6.8 \mathrm{~N} \cdot \mathrm{m}$, producing a force of $85 \mathrm{~N}$. The robot has an electric steering mechanism on each front wheel and an electric driving mechanism on each rear wheel. The total mass of the robot is $110 \mathrm{~kg}$, sprung mass $75 \mathrm{~kg}$ and unsprung mass $35 \mathrm{~kg}$. The robot has sensors: four potentiometers for detecting the suspension displacements, two servo inclinometers for the body attitude, and three rate gyroscopes for the body angular velocity. A computer PC-9801vx with CPU 80286 and NDP 80287 reads all the sensors and controls the currents of the motors.

With the four-wheeled robot, the body attitude in the pitch and roll directions as well as the body height should be controlled. Among these three variables, we started with the two variables of the body attitude.

The state of the robot, that is the body attitude $y$ in this case, has to be detected. We tried using the inclinometer output directly as the attitude of the robot body, or the first order integral of the rate gyro output. We found neither methods very successful, for the inclinometer gave false ripples when the body swung and the gyro integral would drift easily. We devised a filter which combines the inclinometer and the gyro signals to produce a better estimate of the body attitude. The inclinometer output $y_{\mathrm{i}}$ and the rate gyro output $y_{\mathrm{g}}$ are expressed by the following equations in Laplace form if there were no disturbances

$$
\begin{aligned}
& Y_{\mathrm{i}}=Y \\
& V_{\mathrm{g}}=s Y
\end{aligned}
$$

The estimated attitude $y_{\mathrm{e}}$ is calculated by the filter as follows. 


$$
Y_{\mathrm{e}}=Y_{\mathrm{i}} /(T s+1)+V_{\mathrm{g}} T /(T s+1)
$$

where $T$ is a time constant to be determined experimentally. This method is to give greater weight to the inclinometer signal in the lower frequency domain and to the gyro integral in the higher
frequency domain. Using this filter we obtained an accurate estimate of the attitude

to be constant against the disturon robot body attitude value is used for Prsturbance given to the wheels. In the control system, the estimated against the windup. The rate gyol. Its accumulative sum was used for I control with the precaution PID control with the attitu gyro signal was directly used for D control. The block diagram of the the computer. The control cycle was 30 is shown in Figure 4. It was implemented digitally using is, they were taken greatest as far as no ms. The PID gains were determined experimentally, that

me system.

estimation control of the pitch and roll attitude of the robot body, we used two sets of attitude basedion filters each using an inclinometer and a rate gyro. Each suspension was PID controlled combination of the pitch and roll angles. controlling parameters is essentially two.

For the running experiments we made a model rough road. The peak-to-peak value of its profile is $120 \mathrm{~mm}$. We ran the robot over the rough road at a speed of $0.18 \mathrm{~m} / \mathrm{s}$ as shown in Figure 3. Figure 5 (a) shows a result of the suspension control. For comparison, results with no control with fixed suspensions and with free suspensions are shown in Figures 5 (b) and (c). From the diagrams. we see that the maximum attitude deviation is about $\pm 3^{\circ}$ in the control case and much greater in the no control cases. These results clearly show the effectiveness of the control.

\subsection{Roughness clearance}

Besides the body stabilization, it is our great concern how high roughness of a ground a robot can pass over. We made simulations to compare the heights of a step cleared by wheeled robotic vehicles with different types of suspensions. A one-wheel model of a vehicle was considered. In the simulation the vehicle proceeds and hits against a step and succeeds or fails in clearing it. No consideration was made of the driving force, pitching of the body, or any kinds of damping. The active suspension is assumed to control the body height to be constant. Figure 6 shows the three types of vehicle and suspension models and the maximum height of step each vehicle can pass over. We see that an active suspension gives a vehicle clearance of higher steps
than a passive suspension or no suspension.

\section{WHEELED INVERTED PENDULUM}

The estimation of the robot attitude in the gravity space is critical for the dynamic control of a mobile robot. We propose to use an observer which is constructed, based on the modern control theory, using the knowledge of the robot dynamics and the information from internal sensors. We was estimated with the a wheeled inverted pendulum [9]. The attitude, as well as the ground slope, rough road.

\subsection{Estimation of the attitude}

For the control of a dynamic mobile robot, the attitude in the gravity space is an important state of the robot. Usually the attitude is difficult to detect by simply using the signals from sensors. For example, an external sensor contacting the ground gets disturbances from the roughness of the ground; the integration of a rate gyro signal has the problem of drift; an inclinometer does not indicate the direction of gravity when motion acceleration exists. To solve with an observer considering the robot dynamics and the attitude of a mobile robot is estimated internal sensors. robot is expressed

Figure 7 shows the block diagram of the control of a mobile robot. The dynamics of the
expressed

$$
\dot{\boldsymbol{x}}=A \boldsymbol{x}+B \boldsymbol{u}
$$


where $\boldsymbol{x}$ is the state vector of the robot, $\boldsymbol{u}$ is the control vector, and $A$ and $B$ are matrices to describe the dynamics. From the system we can sense the output vector $y$ which is expressed

$$
\boldsymbol{y}=C \boldsymbol{x}
$$

where $C$ is the output matrix.

The observer part is included by the dashed line in Figure 7, where $K$ is the feedback matrix. By selecting proper values for $K$ the loop gets stable and the estimate of the state $x^{*}$ converges to the real state $x$. Then, the feedback matrix $F$ is properly selected to stabilize the total control system using the estimate from the observer.

3.2 Control of the wheeled inverted pendulum

We applied the proposed method to a wheeled inverted pendulum shown in Figure 8 as an example of a dynamic mobile robot. The pendulum has a body and two coaxial wheels which are example of a dynamic mobile robot. The pendulum driven by a DC servo motor contained in the
mounted at one end of the body and rotate together dre
body. The sensors are: a rotary encoder for the motor and a rate gyro on the body.

For the control of this pendulum, we get the angle of the wheel rotation from the encoder and the angular velocity of the body inclination from the rate gyro. We get the angular velocity easily by differentiating the wheel rotation. Now we need to know the angle of the body inclination and for that we use the observer. In this case the observer is fourth order.

The control system was implemented digitally using a computer PC-9801vm with CPU 8086 and NDP 8087. The control cycle was $3 \mathrm{~ms}$. We found that the control was distorted by the nonlinearities of the system: the insensitivity zone and the friction of the driving system. So we inserted the compensation algorithm for the nonlinearities. In the actual system, $\boldsymbol{u}$ and $\boldsymbol{u}^{\prime}$ are onedimensional: the motor current. The estimation of the attitude was made with a good accuracy and the control of stable running of the pendulum on a flat level plane worked successfully.

3.3 Estimation of the slope

For the rough road negotiation of a mobile robot, we want to know the changing slope of the road. We introduced the slope gradient $e$ as a new variable of the state vector $x$. We assumed the dynamics of $e$ as

$$
\dot{e}=0
$$

The dynamics of the pendulum is a little more complicated with the new element $e$ and the observer is fifth order in this case.

Using the observer to estimate the slope, we realized running control of the pendulum on an unknown rough road as shown in Figure 8. Thus, the effectiveness of the proposed method was demonstrated experimentally.

\section{JUMPING ROBOT}

For a wheeled robot to overcome a discontinuous step of the ground, storing energy in springs and releasing it to jump is a possible way. We analyzed the mechanism and fabricated and experimented a wheeled machine that can run and jump.

4.1 Models and analysis

Figure 9 shows some of the possible models of jumping machines using springs which we are going to analyze. The one-spring model (a) consists of a weight $m_{1}$, a frame $m_{2}$, and a spring $k_{1}$ to connect them. The frame is placed on a rigid floor. If we pull the weight down by a travel $s$ and release it, the weight, accelerated by the spring, will go up to collide with the frame. The collision causes the frame to jump. Here we assume:

(1) No energy loss occurs, such as frictional loss, except at the collision.

(2) The coefficient of restitution at the collision of the weight and the frame is zero, which means they move together after the collision.

Denoting $N$ the initial spring force, the maximum height the frame attains after the collision $h$ is expressed 


$$
h=m_{1} s\left(k_{1} s+2 N-2 m_{1} g\right) / 2 g\left(m_{1}+m_{2}\right)^{2}
$$

If we design $N=m_{1} g$

$$
h=m_{1} k_{1} s^{2} / 2 g\left(m_{1}+m_{2}\right)^{2}
$$

The two-spring model (b) is proposed to reduce the energy loss with the one-spring model which occurs at the collision. It is made by adding a spring $k_{2}$ at the bottom of the frame. The maximum attained height $h$ is solved numerically in this case. In the design, given $m_{1}, m_{2}$ and $k_{1}$, we want $k_{2}$ which maximizes $h$. Normalization of parameters reduces the problem: given $\mu=$ $m_{2} / m_{1}$, to optimize $\kappa=k_{2} / k_{1}$. Figure 10 shows the movements of the frame and the weight before the collision for the optimal $\kappa$ for $\mu=0.25$ and 0.75 . The circle indicates the collision with the $\kappa$ and $h$ values in the parentheses. If $\mu \leq 0.75$, we can meet the speeds of the frame and the weight at the collision to allow an efficient jump. However, this is not the case if $\mu \geq 1$.

\subsection{Experiment}

According to the one-spring model we built a wheeled jumping robot shown in Figure 11 with $m_{1}=4 \mathrm{~kg}, m_{2}=3 \mathrm{~kg}$ and $k_{1}=2900 \mathrm{~N} / \mathrm{m}$. Equation (8) gives $h=0.189 \mathrm{~m}$. In the experiment, however, the robot jumped just as high as $0.10 \mathrm{~m}$. The robot made standing jump, running jump and jumping over an obstacle.

\section{CONCLUSION}

We made experiments with an active suspension four-wheeled robot, a wheeled inverted pendulum, and a jumping robot. Emphasis is placed on dynamic control of unconventional mechanisms and attitude estimation using internal sensors. Refining and combining these technologies, we hope to realize a wheeled robot which can negotiate most of uneven grounds.

\section{ACKNOWLEDGEMENT}

We thank Seiichi Usui of the Tokyo Metropolitan Institute of Technology, Shinji Hirobe of the Forestry and Forest Products Research Institute, and Ryohei Matsumoto, Hiroshi Katoh and Kenji Mitsuhashi of Suzuki Motor Corporation, for their advice and contribution to the active suspension experiments.

\section{REFERENCES}

[1] D. Cho, J. K. Hedrick, "Pneumatic actuators for vehicle active suspension applications", ASME J. Dynamic Systems, Measurement and Control, vol. 107, no. 1, pp. 67-72, 1985.

[2] J. Dominy, D. N. Bulman, "An active suspension for a Formula One Grand Prix racing car", ASME J. Dynamic Systems, Measurement and Control, vol. 107, no. 1, pp. 73-78, 1985.

[3] H. G. Gibson, K. C. Elliot, S. P. E. Persson, "Side slope stability of articulated-frame logging tractor", J. Terramechanics, vol. 8, no. 2, pp. 63-79, 1971.

[4] A. Fukuda, K. Ichihara, K. Asahi, M. Mozuna, "The automatic attitude control of hillside tractor for forestry use", J. Japanese Forestry Society, vol. 72, no. 3, pp. 239-246, 1990 (in Japanese).

[5] T. Ohmichi, T. Ibe, "Development of vehicle with legs and wheels", J. Robotics Society of Japan, vol. 2, no. 3, pp. 244-251, 1984 (in Japanese).

[6] N. Kimura, T. Kamigaki, N. Suzuki, A. Nishikawa, N. Yamamoto, "Locomotion mechanism and its control architecture for disaster preventing robot", Proc. '91 International Symposium on Advanced Robot Téchnology, pp. 375-380, 1991. iile.

[7] K. Tani, S. Usui, E. Horiuchi, N. Shirai, S. Hirobe, "A computer-controlled active suspension for a wheeled terrain robot", International J. Computer Applications in Techinology, vol. 3, no. 2, pp. 100-104, 1990.

[8] K. Tani, N. Shirai, H. Katoh, "Active suspension four-wheel model for a terrain robot", Proc. IEEE/RSJ International Workshop on Intelligent Robots and Systems '89, pp. 408-413, 1989.

[9] O. Matsumoto, S. Kajita, K. Tani, "Estimation and control of the attitude of a dynamic mobile robot using internal sensors", J. Robotics Society of Japan, vol. 8, no. 5, pp. 541-550, 1990. 


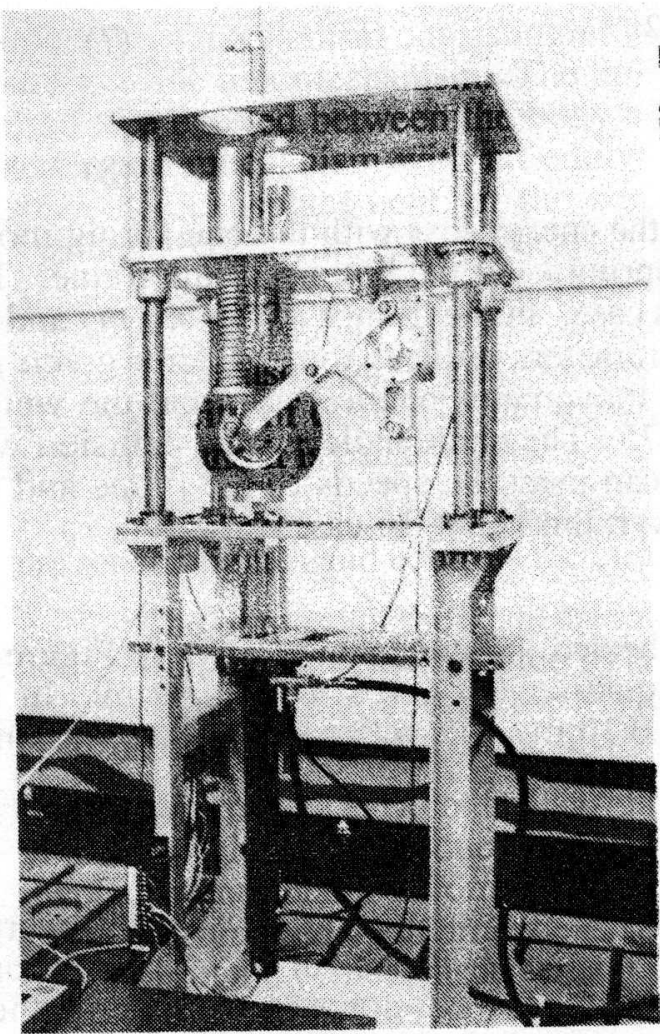

Figure 1 Active suspension one-wheel model.

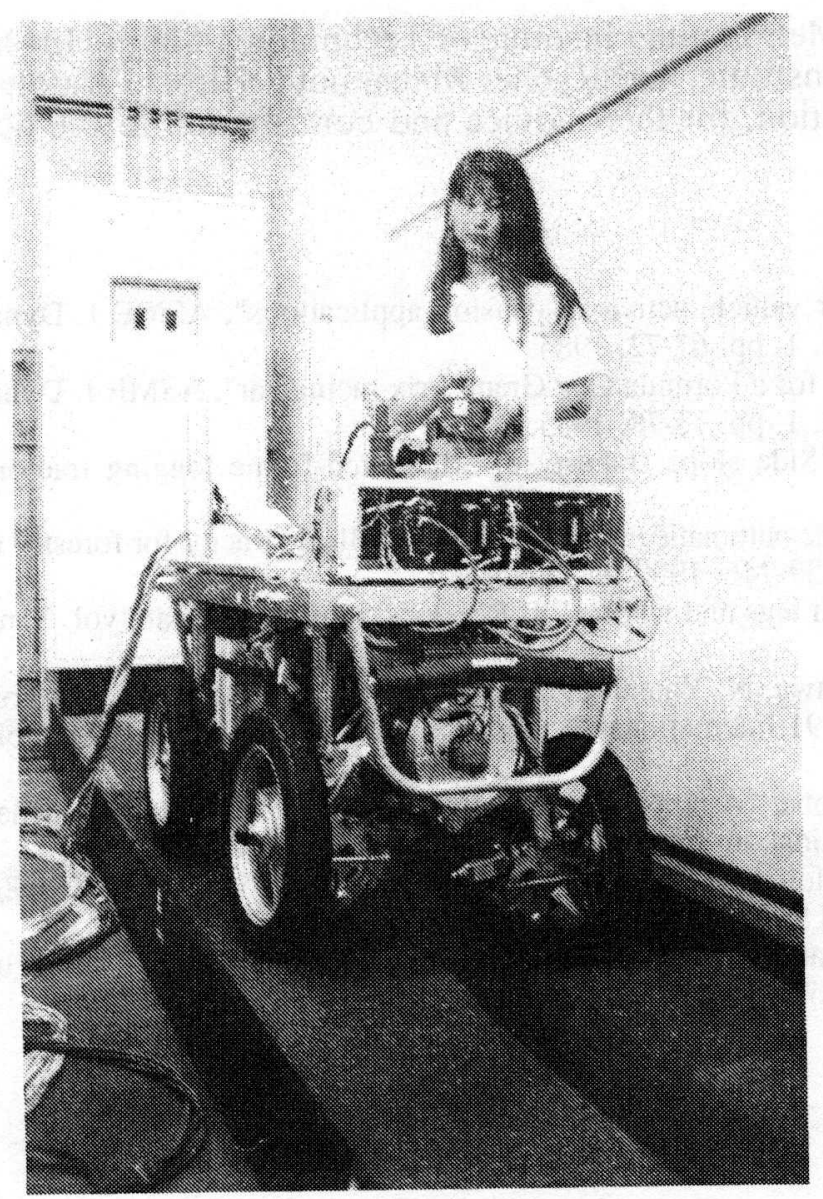

Figure 3 Active suspension four-wheeled robot running over the model rough road.
Disturbance Road Inclination is

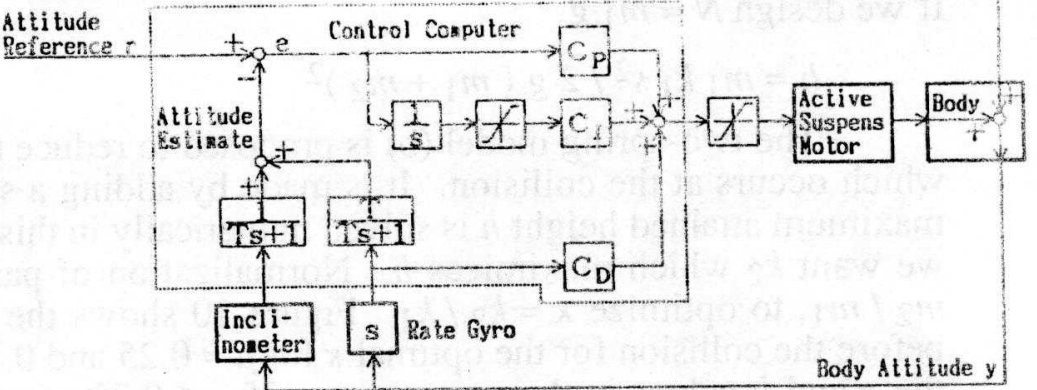

Figure 4 Block diagram of PID control of the active suspension for the body attitude regulation with the attitude estimation by filtering the inclinometer and the gyro signals.

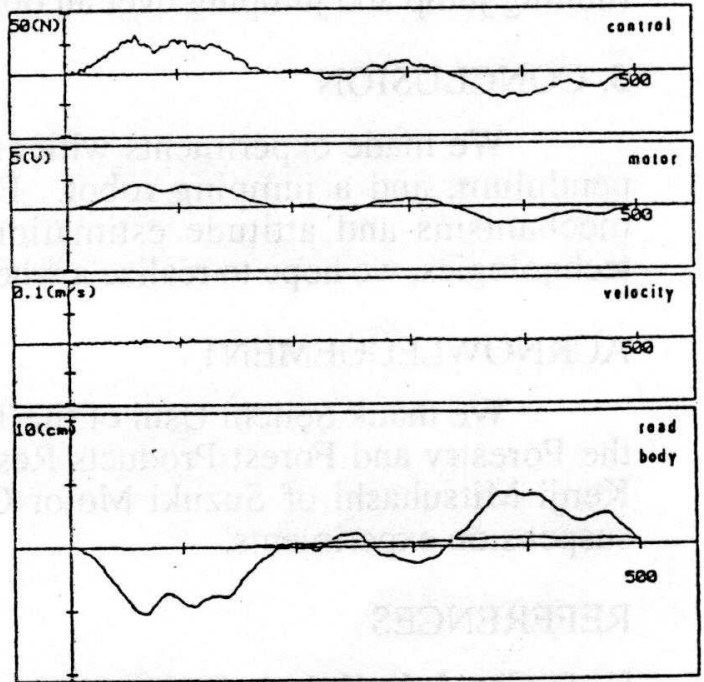

(a) PI control using motor displacement and body velocity.

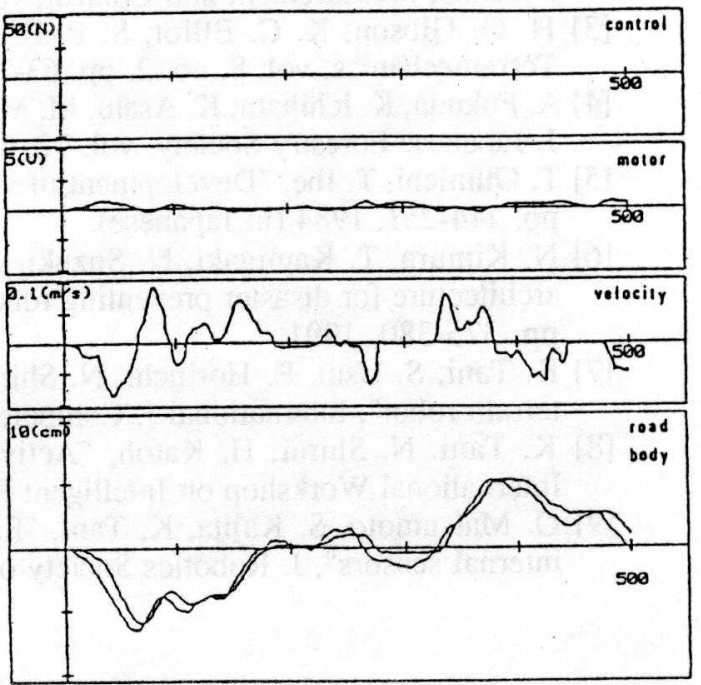

(b) No control.

Figure 2 Experimental results of the active suspension one-wheel model. 

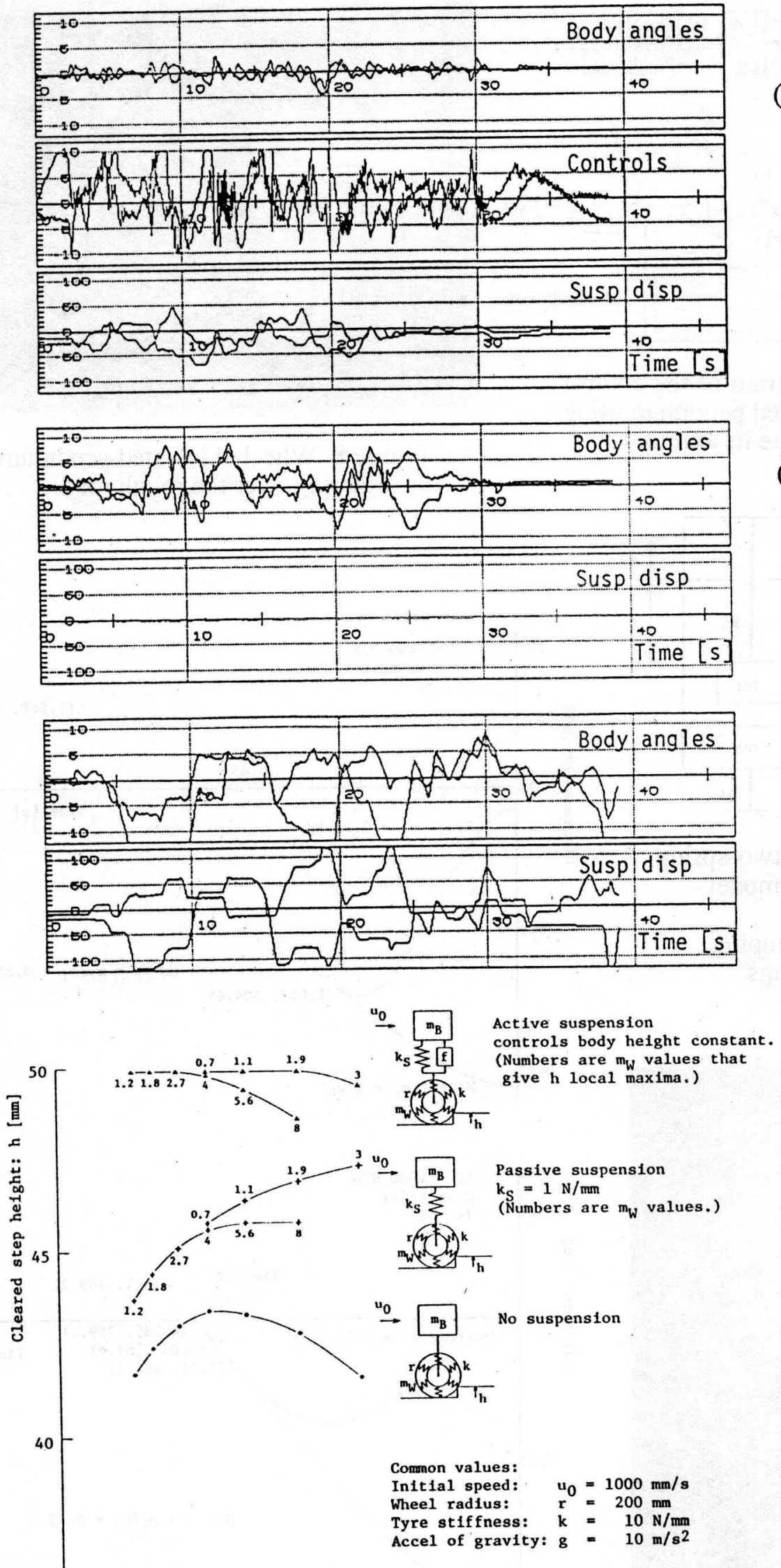

(a) PID control of the suspensions.

(b) No control with fixed suspensions. (c) No control with free suspensions.

Figure 5 Experimental results of the active suspension fourwheeled robot running over the model rough road.
35

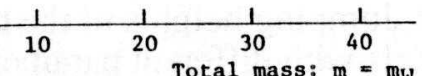

Figure 6 Step heights cleared by vehicles with different suspensions. 


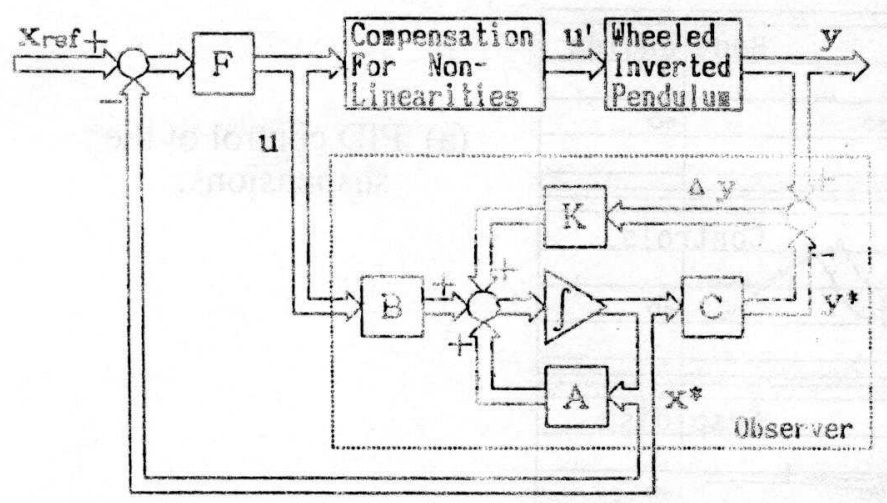

Figure 7 Block diagram of the control of the wheeled inverted pendulum using an observer to estimate its attitude.

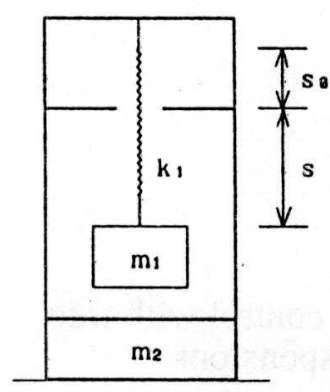

(a) one-spring model

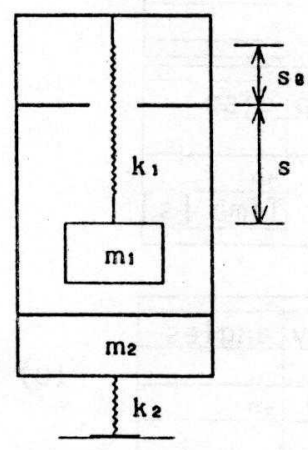

(b) two-spring model

Figure 9 Models of jumping machines using springs.

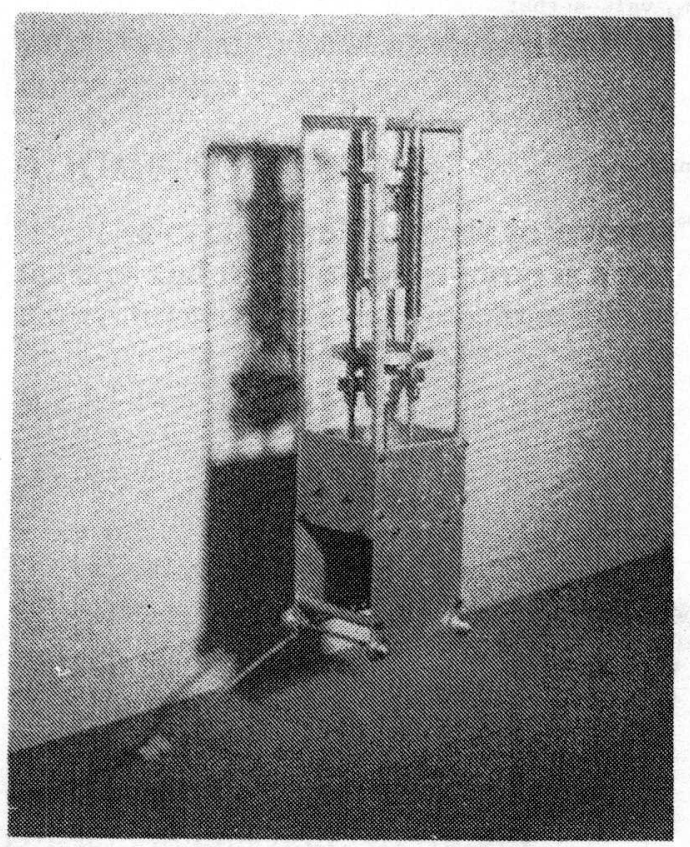

Figure 11 Wheeled jumping robot.

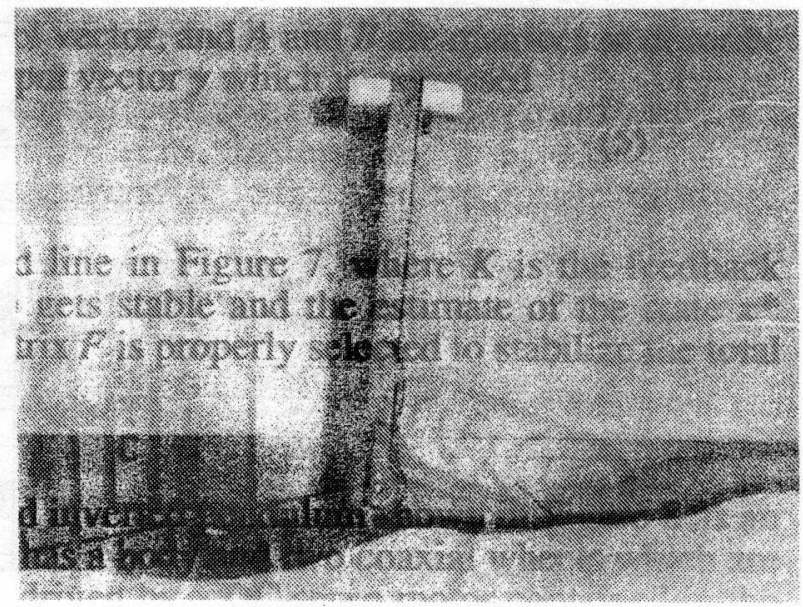

Figure 8 Wheeled inverted pendulum running over the rough road.
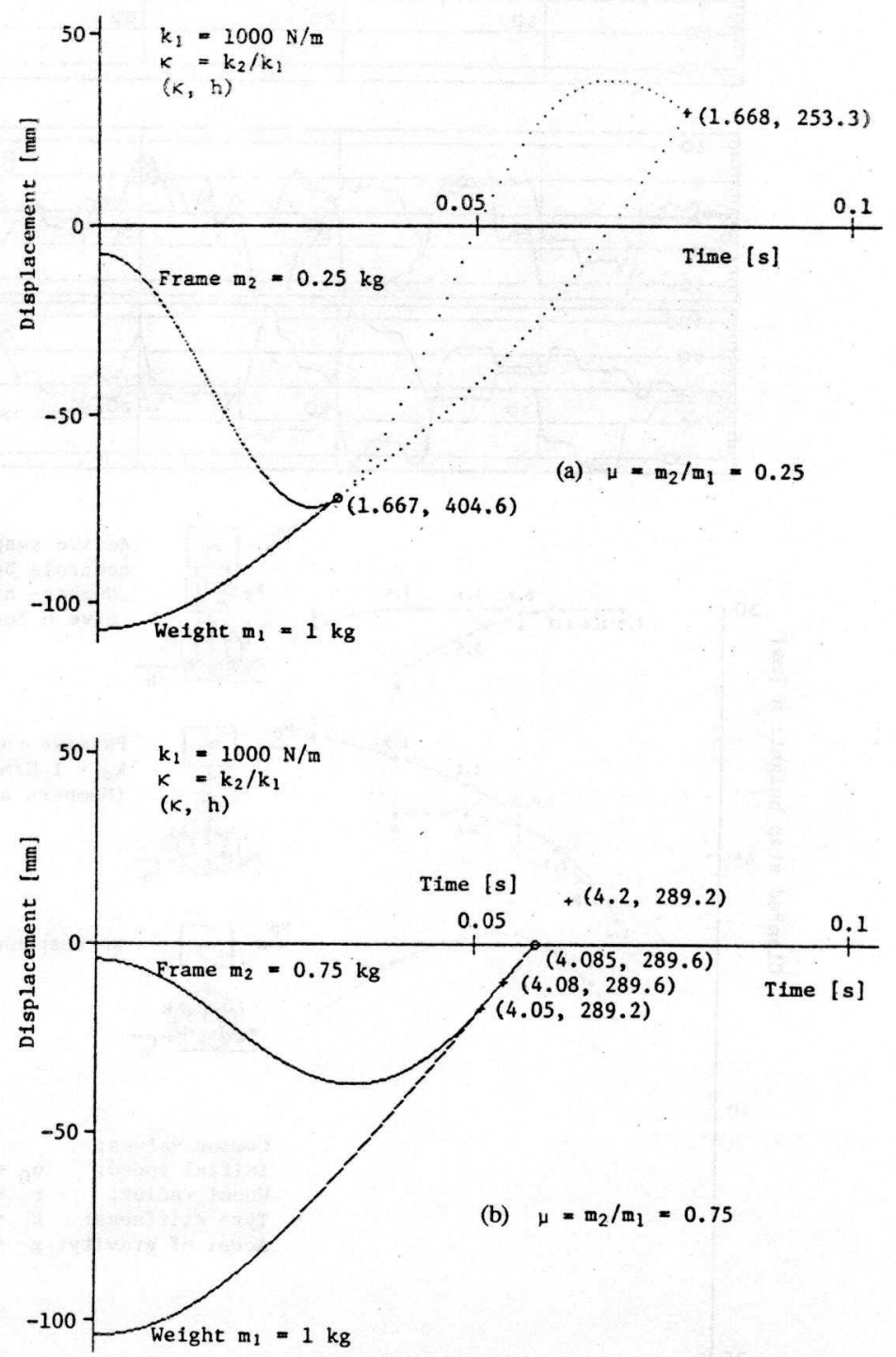

Figure 10 Jumping heights of the two-spring models with different parameters. 